University of Nebraska - Lincoln

DigitalCommons@University of Nebraska - Lincoln

1982

\title{
The Bead Game: Response Strategies in Free Assortment
}

Alan B. Bond

University of Nebraska - Lincoln, abond1@unl.edu

Follow this and additional works at: https://digitalcommons.unl.edu/bioscibond

Part of the Behavior and Ethology Commons

Bond, Alan B., "The Bead Game: Response Strategies in Free Assortment" (1982). Alan Bond Publications. 1.

https://digitalcommons.unl.edu/bioscibond/1

This Article is brought to you for free and open access by the Papers in the Biological Sciences at DigitalCommons@University of Nebraska - Lincoln. It has been accepted for inclusion in Alan Bond Publications by an authorized administrator of DigitalCommons@University of Nebraska - Lincoln. 


\title{
The Bead Game: Response Strategies in Free Assortment
}

\author{
Alan B. Bond \\ Department of Psychology, University of California, Berkeley
}

\begin{abstract}
Subjects were presented with a collection of spherical beads of four different colors and were instructed to sort them as fast and as accurately as possible. The sequence in which the beads were sorted was recorded, along with the time intervals between successive beads. Subjects were observed to sort in nonrandom sequences, producing runs in which a given bead type was taken exclusively. The speed and accuracy of the sorting process was positively correlated with the degree of nonrandomness of the sorting sequence. This relationship appeared to be primarily attributable to perceptual factors involved in the initiation of a run and secondarily attributable to facilitation of sorting movements within runs. The effect of sorting sequence was enhanced with bead colors that were harder to discriminate. The results are interpreted in the light of known effects of presentation sequence and stimulus discriminability on stimulus processing.
\end{abstract}

\section{Introduction}

Free assortment, the segregation of a heterogeneous collection of objects into uniform classes, is a commonplace human task. It is encountered in numerous commerical and industrial applications involving visual classification, such as inventory or hand assortment of coins, electronic parts, and hardware, or inspection tasks that require grading or separation of different classes of defective items. Free assortment differs from most other experimental preparations in cognitive psychology in that all of the items in the stimulus array are potential targets, all are presented simultaneously at the start of a trial, and the order of items to be classified is not specified by the experimenter. The lack of a prescribed sorting sequence has consequences that have never been thoroughly explored. In particular, it may allow the subject to employ response strategies to reduce the amount of information processing required and, thereby significantly increase the speed and accuracy of the sorting process.

If assortment is viewed as a concatenated series of single stimulus identifications, the process must involve a serial memory search, in which the given stimulus item is compared successively with the remembered defining features of each class. If the search is self-terminating and the sequence of presentations is nonrandom, containing contiguous sequences of a single stimulus type ("runs"), the subject should be able to reduce processing time and increase response accuracy by initiating the comparison with the salient features of recently presented stimuli. It is well known that repeated stimuli are, in fact, classified more rapidly and more accurately than are randomly ordered stimuli, though the phenomenon is apparently complex, involving motor as well as perceptual com- 
ponents (Rabbitt et al., 1977). It might be expected, therefore, that subjects performing free assortment would elect to sort in nonrandom sequences, and that the efficiency of the sort would prove to be correlated with its degree of nonrandomness.

Facilitation of serial processing may not be the sole advantage of nonrandom sequences. In visual displays with several types of discriminable targets, focusing attention on one target type allows the subject to view it temporarily as the positive set, while the rest are classed as negative (Gottwald and Garner, 1972). Irrelevant items are apparently not identified under these circumstances (Neisser and Beller 1965), and the items in the positive set are processed effectively in parallel. Since the visual distinctiveness of the item categories is a critical factor in parallel processing of visual stimuli (Schneider and Schiffrin, 1977), differences would be expected both in sorting efficiency and in the effect of different sorting strategies, depending on the difficulty of the discrimination task involved. To explore the strategies adopted in free assortment and their consequences for sorting efficiency, a schema was developed that allowed automatic recording of the time and sequence of the assortment of a set of colored beads.

\section{Method}

Subjects were asked to sort, by color, a collection of painted wooden spheres about one centimeter in diameter, into target funnels, as fast and as accurately as possible, moving only one bead at a time. To facilitate handling, the beads were placed on a $53 \times 33-\mathrm{cm}$ mat of knobbed brown rubber surrounded by a $1.5-\mathrm{cm}$ rim. For each trial, 100 beads, 25 of each of four colors, were thoroughly mixed and were spread evenly over the sorting surface, so that each bead was separated from its neighbors by roughly two diameters. At the farther edge of the sorting surface, four plastic funnels, $6.4 \mathrm{~cm}$ in diameter, were mounted in a horizontal array with $10 \mathrm{~cm}$ spacing between the centers. The funnels were tilted toward the subject at $30^{\circ}(0.524 \mathrm{rad})$ to the plane of the table and were surrounded with 2-cm-wide collars painted with the same pigments as the beads. As beads were dropped singly into the funnels they activated a microswitch in the stem and fell into register against a background of the correct color, allowing the experimenter to check for errors. The time interval between successive beads was recorded to millisecond accuracy.

The 12 subjects (5 females and 7 males) were all students in an introductory psychology course. They were shown the stimulus beads before the data run and were allowed to familiarize themselves with the color categories. None of the subjects admitted to a history of difficulty in visual discrimination, problems of hand-eye coordination, or extensive prior experience involving assortment. At the end of each trial, they were informed of the sorting time and number of errors for that trial and were given comparison data from their own best previous effort and from the best overall performance by other subjects. Each subject performed 20 sorting trials, 10 on each of two stimulus sets of differing discriminability, in randomized order.

The stimulus sets were prepared from combinations of six different bead colors. Stimulus Types 1 and 6 were painted gloss white and flat black, respectively, with spray enamel. The remaining four stimuli were shades of pastel green, generated by mixing various proportions of pure pigments with a latex enamel base. The Easy task required discriminating beads of types 1, 2, 4, and 6; the Hard task consisted of types 2, 3, 4, and 5. Each pigment was characterized as to brightness, excitation purity (or saturation), and dominant wavelength (or hue), on a spectrophotometer against a barium sulphate standard (Table 1). The stimuli used in each task were approximately equally spaced in log brightness, with the separation in the Easy task being about twice that in the Hard. The colors of the target funnels were ordered by brightness, the direction of the ordering being balanced across subjects. The stimulus array was illuminated from above and in front of the subject by a bank of softwhite fluorescent bulbs. 


\section{Results}

\section{Sorting Sequence}

The expected number of runs in a random ordering of 100 items, 25 of each of four types, is 76 (derived by the method of Brownlee, 1965). By this criterion, subjects sorted exclusively in nonrandom sequences, and the mean number of runs in a sorting trial was $20.95 \pm 2.33$, significantly less than the random expectation. A two-way analysis of variance (task $\times$ subject) revealed no effect of task difficulty on number of runs, $F(1,216)=1.12, p>0.2$, but did reveal significant differences between subjects, $F(11,216)=65.6, p<0.001$, with nearly $70 \%$ of the overall variance being attributable to betweensubject differences.

Further analysis was greatly simplified by dividing the 12 subjects into three groups of four on the basis of the characteristic number of runs they produced. The Low group averaged between 4.5 and 8.2 runs per trial (group mean $=6.33$ ). The range for the Medium group was 13.3 to 23.3 (mean = 17.4), while that for the High group was 30.4 to 58.9 (mean $=39.1)$. Because the grouping made use of natural breakpoints, it produced only a negligible loss of information: $79 \%$ of the between-subjects variance in number of runs was retained in the grouped data. Sex was not a significant factor in the differences between subjects, and the sexes were evenly distributed among the three groups.

\section{Sorting Efficiency}

The total time for the sort exhibited a practice effect (i.e., a negatively accelerated, monotonic decrease with trial sequence) for both task types. Multivariate regression of sorting time on trial sequence and the square root of the trial sequence yielded a correlation of 0.57 for the Easy task $(p<$ $0.001)$ and 0.67 for the Hard task $(p<0.001)$. Practice effects therefore accounted for 30 to $40 \%$ of the overall variance. There was no effect of trial sequence on number of runs, however $(r=0.23, p>$ 0.1 ), implying that the decrease in sorting time with practice probably reflected an increased familiarity with the demands of the task, rather than a systematic change in sorting strategy. The time inter-

Table 1. Characterization of Stimuli

\begin{tabular}{lrrr}
\hline $\begin{array}{l}\text { Stimulus } \\
\text { Number }\end{array}$ & Brightness & $\begin{array}{r}\text { Dominant } \\
\text { Wavelength (nm) }\end{array}$ & $\begin{array}{r}\text { Excitation } \\
\text { Purity (\%) }\end{array}$ \\
\hline 1 & 83.5 & 538 & 0.63 \\
2 & 43.7 & 536 & 15.57 \\
3 & 26.0 & 528 & 10.44 \\
4 & 15.4 & 508 & 6.03 \\
5 & 9.6 & 495 & 5.86 \\
6 & 4.7 & 477 & 3.57 \\
\hline
\end{tabular}

Hard Task: Stimuli 2, 3, 4, and 5.

Easy Task: Stimuli 1, 2, 4, and 6. 
vals between successive beads ("transfer times") were therefore corrected for practice effects by multivariate regression within subjects against the trial sequence variables. Residuals from this regression were used to generate corrected values for the transfer times, which were then summed within each trial to compute a corrected sorting time.

The results of a two-way analysis of variance (task $x$ run group) on the corrected sorting time, summarized in Table 2A, confirm that subjects who sorted in longer, less numerous runs also required less time, $F(2,234)=33.3, p<0.001$. The Hard task required about $7 \%$ more time to sort, and this difference was also significant, $F(1,234)=21.6, p<$ 0.001 . There was no significant interaction of run group with task type for sorting time, $F(2,234)=$ $1.00, p>0.3$.

Error rates were very low for both task types, amounting to less than $0.3 \%$ in the Easy task and $1.7 \%$ in the Hard task (Table 2B). Analysis of variance confirmed the significance of this difference, $F(1,234)=32.1, p<0.001$. There was a significant main effect of run group on the error rate, with the highest accuracy being associated with the longest runs, $F(2,234)=9.32, p<0.001$. The interaction was also significant, $F(2,234)=6.18, p<0.002$, implying that the effect of run group on accuracy was stronger in the Hard task.

Table 2. Sorting Efficiency

\section{A. Mean Corrected Sort Time}

\begin{tabular}{lcccc} 
& \multicolumn{4}{c}{ Number of Runs } \\
Task & Low & Med & High & Mean \\
\hline Easy & 79.32 & 86.46 & 91.63 & 85.80 \\
Hard & 83.54 & 95.28 & 97.45 & 92.09 \\
Mean & 81.43 & 90.87 & 94.54 &
\end{tabular}

B. Mean Number of Errors per Trial

\begin{tabular}{lcccc} 
& \multicolumn{4}{c}{ Number of Runs } \\
Task & Low & Med & High & Mean \\
\hline Easy & 0.125 & 0.350 & 0.325 & 0.267 \\
Hard & 0.350 & 2.650 & 2.000 & 1.667 \\
Mean & 0.238 & 1.500 & 1.163 & \\
\hline
\end{tabular}

\section{Analysis of Individual Bead Transfers}

Given the transfer times for each individual bead, it was possible to conduct a post hoc analysis of the sorting time in an effort to determine the source of the increased efficiency. The time interval between successive beads within a run of a single color (Same transfers) was found to be significantly smaller, $t(23645)=17.1, p<0.001$ than the time between beads of different colors (Different transfers), the mean difference being about $50 \mathrm{~ms}$ for the Easy task and $180 \mathrm{~ms}$ for the Hard task (Table 3). All bead transfers were significantly slower in the Hard task (for Same transfers, $t(18858)=5.64, p<0.001$; for Different, $t(4785)=$ 9.71, $p<0.001)$, but the difference between transfer types was proportionately greater, $F(1,23635)$ $=16.5, p<0.001)$, a matter of $18 \%$, as opposed to $4 \%$ in the Easy task.

Same transfers. The effect of run group on transfer time for Same transfers was examined with a two-way (task $x$ run group) analysis of variance (Table 4A). There was a significant effect of number of runs, $F(2,18854)=188, p<0.001$, in addition to the task effect noted earlier. There was no significant interaction, however, $F(2,18854)=1.75$, $p>0.15)$, implying that the decrease in Same transfer time with number of runs was independent of task difficulty.

Table 3. Mean Transfer Time (ms)

\begin{tabular}{|c|c|c|c|}
\hline \multirow[b]{2}{*}{ Task } & \multicolumn{3}{|c|}{ Transfer Type } \\
\hline & Same & Different & Mean \\
\hline Easy & 844 & 892 & 853 \\
\hline Hard & 874 & 1055 & 912 \\
\hline Mean & 859 & 976 & \\
\hline
\end{tabular}


Table 4. Mean Transfer Time (ms)

\section{A. Same Transfers}

Number of Runs

\begin{tabular}{lcccc} 
Task & Low & Med & High & Mean \\
\hline Easy & 791 & 853 & 914 & 844 \\
Hard & 810 & 895 & 946 & 874 \\
Mean & 800 & 873 & 930 & \\
\hline
\end{tabular}

\section{B. Different Transfers}

Number of Runs

\begin{tabular}{lrrrr} 
Task & Low & Med & High & Mean \\
\hline Easy & 759 & 899 & 907 & 892 \\
Hard & 1120 & 1115 & 1017 & 1055 \\
Mean & 946 & 1020 & 962 & \\
\hline
\end{tabular}

The relevance of this result to investigation of the sorting mechanism became more apparent when the individual transfer times for Same transfers were pooled across subjects and recategorized according to the length of the preceding homogeneous run (between 1 and 24). Means of the runlength categories for each task type are plotted in Figure 1. A polynomial regression analysis of these data yielded strong positive correlations between transfer time and run length for both task types $(r$ $=0.90$ for the Easy task and 0.87 for the Hard task), with a significant quadratic component $(F(1,21)$ $=5.65, p<0.03$ for the Easy task; $\mathrm{F}(1,21)=19.2, p$ $<0.001$ for the Hard task). Analysis of covariance showed no significant inhomogeneity in the slope estimates, $F(2,42)=0.129, p>0.3$, implying that the shapes of the curves for the two tasks were not statistically distinguishable, and that the effect of task difficulty was simply to alter the y-intercept. Bestfitting slope estimates were, therefore, derived by pooling across tasks, and the resulting curves are plotted in Figure 1.
Different transfers. The effect of run group on the speed of Different transfers (Table 4B) was marginally significant, $F(2,4781)=3.78, p<0.03$, but it interacted strongly with the difficulty of the sorting task, $F(1,4781)=24.8, p<0.001$. To elucidate the nature of the interaction, one-way analyses of variance were performed on each task separately. In the Easy task, there were significant differences between run groups, $F(2,2318)=15.2, p<0.001$, with Different transfers in the Medium and High groups requiring significantly more time, $t(2318)=5.36, p<$ 0.001. In the Hard task, the significant effect of run group, $F(2,2463)=5.48, p<0.005$, was mainly attributable to the reduction in Different transfer time in the High group, $t(2463)=3.00, p<0.003$.

If the bead chosen in a Different transfer represented a random selection from the remaining beads (or at least those that differed in color from the preceding run), the relationship between the probability of a switch to any given color and the proportion of that color remaining on the table would be linear, with a slope of 1 . If, on the other hand, subjects tended to concentrate, when starting a new run, either on the most common color remaining or on the least common, this behavior should be detectable as a systematic deviation from the predicted linear relationship. To generate these curves, the random probabilities of each possible color choice were calculated for each Different transfer, excluding the color of the preceding run. The observed probability of selection was then calculated for each of 20 equal intervals along the random probability axis. Data segregated by task type and run group are displayed in Figures 2 and 3, respectively. Overall, there was a significant deviation from a random-selection expectation, $\chi^{2}(19)=10,265, p<0.001$, most of it being attributable to over-selection of the less frequent colors. The tendency to concentrate on rarer types was significantly related to run group, $\chi^{2}(33)=1,259, p<$ 0.001 , with the largest deviation occurring in the Low group. There was also a significant task effect, with greater deviation occurring in the Hard task, $\chi^{2}(19)=105, p<0.001$. 


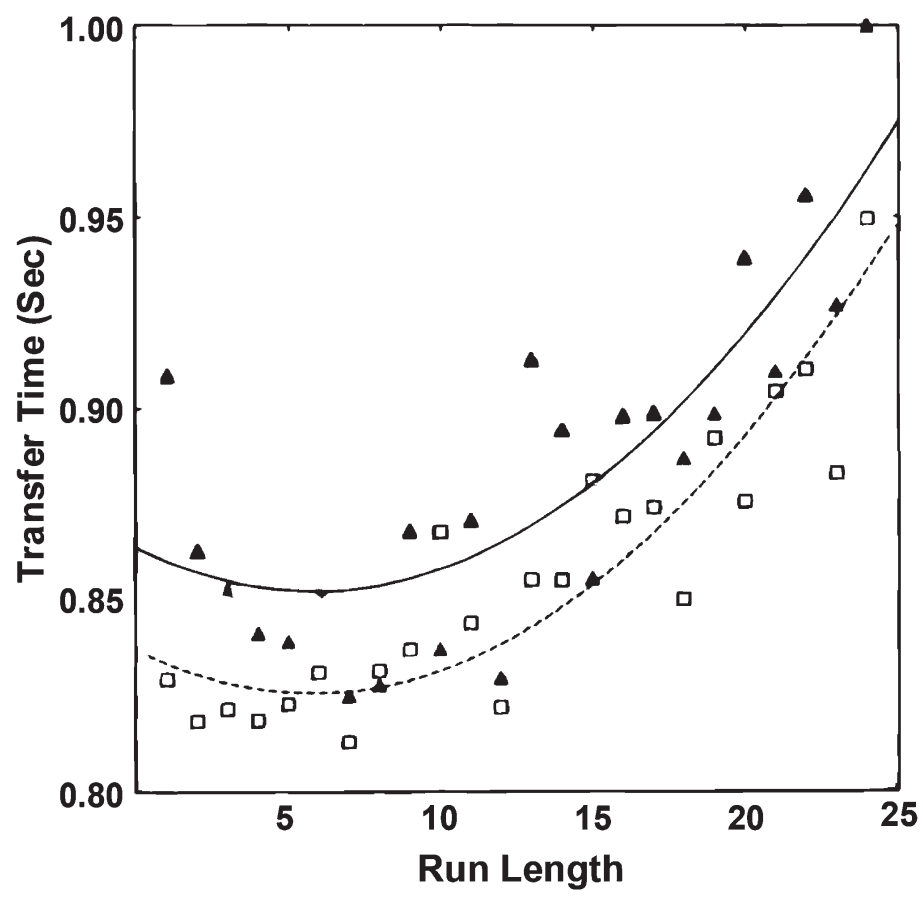

Figure 1. Mean duration of Same transfers as a function of the length of the preceding homogeneous run. Dashed line and squares indicate Easy Task; solid line and triangles indicate Hard Task.

\section{Discussion}

\section{Sorting Sequence and Efficiency}

Although all of the subjects invariably sorted in nonrandom sequences, those who produced longer, less numerous runs were noticeably more efficient. Long-run subjects required $16 \%$ less time to complete a sorting trial than their short-run colleagues and exhibited only $20 \%$ of the error rate (Table 2). There was no indication that subjects improved their response strategies in the course of the experiment. However, the practice effect in the first 20 trials was large enough that it may have prevented them from being able to evaluate the consequences of varying the sorting sequence. Given the lack of an a priori reason to expect a coincidental association between dexterity and nonrandomness, it seems reasonable to interpret the results as causal effects of sorting sequence and target discriminability on stimulus processing.
The main effect of task type on sorting time (Table 2A) is scarcely surprising. That high target/background similarity can increase response time has been observed in studies dating back to Neisser (1963). The interaction between task and run group for error rate (Table 2B), however, suggests that there may be qualitative differences between tasks, as well. Errors in the Easy task may be different in kind from those made when the targets are harder to discriminate (Wilding, 1971). Estes (1972) has claimed that errors made with a non-confusable background do not represent incomplete or inaccurate processing, but rather lapses in the mechanism of response assignment. If the Hard task elicited both types of error, while the Easy task produced only the latter, the proportionate increase in error rate for a given decrease in run length would be higher for the Hard task, thus accounting for the interaction. 


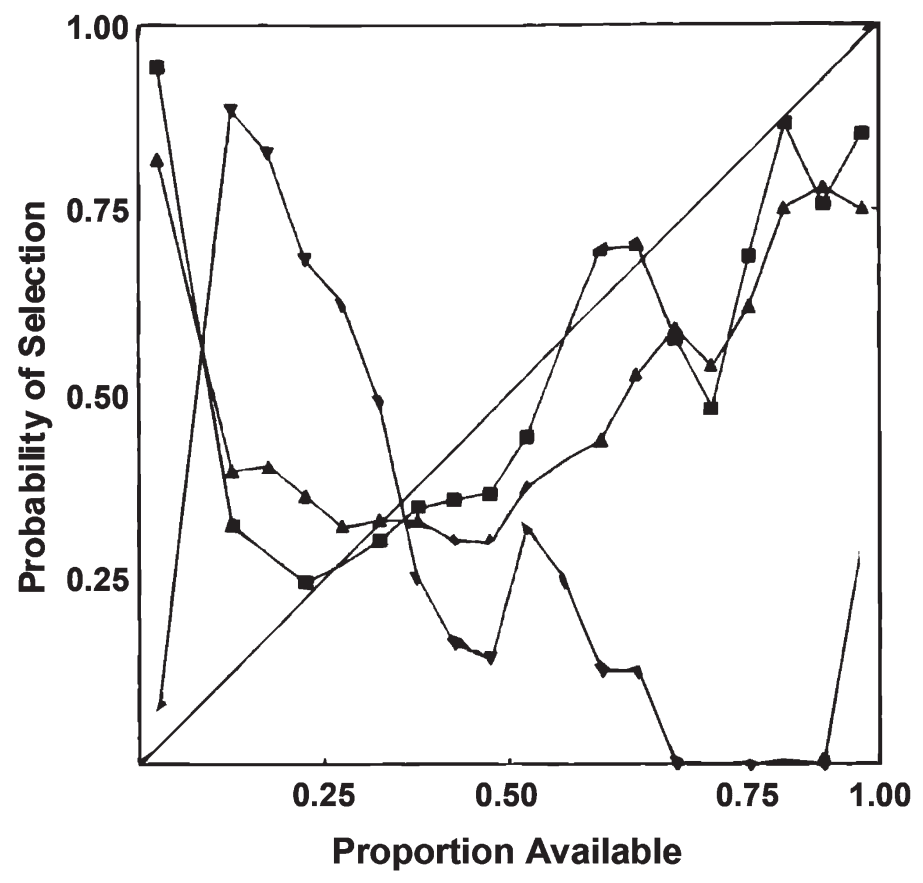

Figure 2. Probability of selecting a given bead color in a Different transfer, as a function of the proportion of that color remaining. Squares indicate High run group; erect triangles indicate Medium run group; inverted triangles indicate Low run group. Graphs have been smoothed once by the method of running means.

The effect of run length on sorting efficiency probably involves both motor and perceptual factors. Since the beads were categorized by sorting them into separate physical locations, transferring beads of different colors required slightly different hand movements. When the number of response alternatives is large, the decrease in response time consequent on making repeated, identical movements is considerable (Kornblum, 1973). One would therefore expect a substantial motor component to the effect, assuring that Same transfers should be faster than Different transfers, irrespective of the perceptual demands of the task. The significantly greater difference between Same and Different for the Hard task (Table 3), however, suggests that some perceptual effects must also be operating.

\section{Analysis of Same Transfers}

The contributions of the perceptual and motor components can be separated by close analysis of the Same transfers (Figure 1). With increasing run length, Same transfers decreased to a minimum at a run length of about 6 . The subsequent increase probably reflects an increase in searching time as the number of beads of the given color is reduced. Because there was no significant interaction between task and run length for Same transfers (Table $4 \mathrm{~A}$ ), the initial decrease can be interpreted as reflecting only the motor component of the repetition effect. The intercept of the Same curve would then constitute an estimate of the predicted time for Different transfers if the motor component were all that was involved. The intercepts are clearly lower 


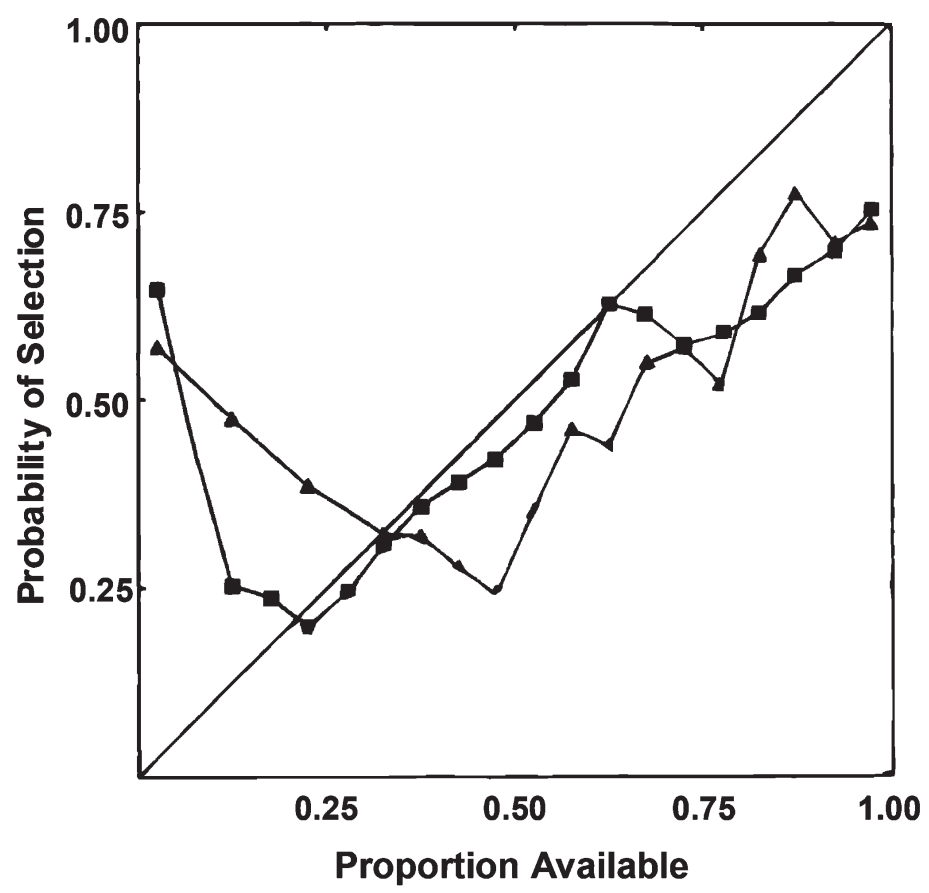

Figure 3. Probability of selecting a given bead color in a Different transfer, as a function of the proportion of that color remaining. Squares indicate Easy task; triangles indicate Hard task. Graphs have been smoothed once by the method of running means.

than the observed mean values for Different (837 vs. 892 for Easy; 863 vs. 1055 for Hard). This suggests that perceptual effects in free assortment are most evident in the contrast between Different and Same transfers, and that they contribute little to the consequences of additional repetitions of the same stimulus.

The only task effect on Same transfers was to offset the transfer time by a constant amount (about 30 $\mathrm{ms})$, an expression of the additional time required to discriminate the next item of a particular color in the Hard task environment. This may reflect a difference between tasks in the ability of subjects to process the items in parallel. Focusing attention on one bead type at a time presumably allows the subject to detect the next bead to be sorted peripherally during the transfer ofthe previous bead. Since the effect of attention on peripheral acuity appears to be reduced in more difficult discriminations (Mackworth, 1965; Beck and Ambler, 1973), this technique may be less effective in the Hard task, and the sub- ject may have to resort more often to controlled, serial search (Schneider and Shiffrin, 1977).

\section{Analysis of Different Transfers}

The repetition effect alone does not predict a main effect of run group on the duration of Different transfers, since the presumed processing economy occurs during runs, rather than between them. Other time components, related to the means by which the sorting sequence is generated, are also involved in Different transfers, however, and may vary significantly from one run group to another. Different transfers entail a determination that there are no more beads of the given color in the region under examination and a consequent decision to switch to another stimulus type. Therefore, they must necessarily include the time required to arrive at this decision. Because of the greater search time involved, longer, less numerous runs would be expected to require proportionately longer deci- 
sion times. This appears to be the case, at least for the Hard task (Table 4B). The shorter decision time for the Low run subjects on the Easy task may indicate that they are scanning the field in peripheral vision and making the decision to switch during the movement of the previous bead. Or, it may simply reflect a greater tendency to systematic search of the display (Clement and Schiereck, 1971).

The selection curves suggest that, by concentrating their efforts on the removal of low frequency beads, the subjects were completely eliminating one bead category at a time from a localized region. It is possible, therefore, to view the differences between run groups (Figure 2) as reflecting differences in the size of an area that is systematically cleared. The Low group uses the entire sorting surface, while the High group concentrates on a fraction of the display at a time. A bias toward selection of infrequent bead types, when added to the primary strategy of taking them in runs, could enhance sorting efficiency by progressively reducing the diversity of nontarget beads. Background heterogeneity has been shown to have a marked effect on response time, particularly when the targets and distractors are difficult to discriminate (Gordon, 1968; Gordon, Dulewicz, and Winwood, 1971; Estes, 1972). Thus, the progressive simplification of the background should tend to reduce sorting time. Several subjects reported that they developed the strategy of removing the most extreme bead types in the Hard task first, thereby reducing the difficulty of the central comparison.

\section{Summary and Recommendations}

The increase in efficiency associated with sorting items in runs appears to result mainly from a reduction in the number of Different transfers $(10 \%$ of the variance in sort time). The mechanism probably involves focusing attention on the features of one stimulus type at a time, thereby reducing the complexity of serial categorization and allowing parallel processing when the stimuli are sufficiently distinct. Differences in Same transfer time as a function of run length (an additional 3\% of the variance) can be attributed to a facilitation of repeated movements and possibly to a progressive reduction in background heterogeneity. The effect of run length on Different transfer time was minimally important. The pattern of selection of stimuli in Different transfers suggested that variation in the sorting sequence was obtained by systematically removing all items from localized regions of different sizes.

For the Easy task, the duration of both Different and Same transfers was found to decrease with increasing run length, suggesting that the optimal strategy in easy discriminations is always to remove all items of a given type in a single run. However, it is entirely possible that the optimum run length in a difficult discrimination might be less than the maximum value, particularly if the number of targets in the display is large. Practitioners who wish to maximize sorting efficiency in an industrial process might consider instructing their subjects (1) to clear a specified proportion of the display at a time, using the longest sorting runs the area will allow, and (2) to first sort those item types that are relatively infrequent or deviant in appearance. The proportion searched could then be varied experimentally over a broad range to discover the optimum run length for the given task conditions.

\section{Acknowledgments}

My thanks to D.A. Riley, S. Sternberg, S.J. Spengler, and R.G. Cook for critical advice and assistance. This research was supported in part by a postdoctoral fellowship from the National Institutes of Mental Health.

\section{References}

Beck, J., and Ambler, B. The effects of concentrated and distributed attention on peripheral acuity. Perception and Psychophysics, 1973, 14, 225-230.

Brownlee, K.A. Statistical theory and methodology in science and engineering. New York: Wiley, 1965.

Clement, D.E., and Schiereck, J.J. Scanning strategies and differential sensitivity in a visual signal-detection task: Intersubject consistency. Psychonomic Science, 1971, 23, 385-387. 
Estes, W.K. Interactions of signal and background variables in visual processing. Perception and Psycho- physics, 1972, 12, 278-286.

Gordon, I.E. Interactions between items in visual search. Journal of Experimental Psychology, 1968, 76, 348-355.

Gordon, I.E., Dulewicz, V., and Winwood, M. Irrelevant item variety and visual search. Journal of Experimental Psychology, 1971, 88, 295-296.

Gottwald, R.L., and Garner, W.R. Effects of focusing strategy on speeded classification with grouping, filtering, and condensation tasks. Perception and Psychophysics, 1972, 11, 179-182.

Kornblum, S. Sequential effects in choice reaction time: A tutorial review. In S. Kornblum (Ed.) Attention and Performance IV. New York: Academic Press, 1973, 259-288.

Mackworth, N.H. Visual noise causes tunnel vision. Psychonomic Science, 1965, 3, 67-68.
Neisser, U. Decision-time without reaction time: Experiments in visual scanning. American Journal of Psychology, 1963, 76, 376-385.

Neisser, U., and Beller, H.K. Searching through word lists. British Journal of Psychology, 1965, 56, 349-358.

Rabbitt, P.M.A., Cumming, G., and Vyas, S. An analysis of visual search: Entropy and sequential effects. In S. Dornic (Ed.) Attention and performance VI. Hillsdale, NJ: Erlbaum, 1977, 363-386.

Schneider, W., and Schiffrin, R.M. Controlled and automatic human information processing: I. Detection, search, and attention. Psychological Review, 1977, 84, $1-66$.

Wilding, J.M. The relation between latency and accuracy in the identification of visual stimuli. I. The effects of task difficulty. Acta Psychologica, 1971, 35, 378-398. 